\title{
Les maladies des voies biliaires intra-hépatiques
}

On a tenement perfectionné Гexploration du cholédoque - cholan-giographie, radiomanométrie per- et post-opératoire, cholédoscopie, etc. - que les voies biliaires supérieures, localisées dans le foie lui-même, ont été singulièrement negligees. Lorsqu'on observe, sur les cliches, une opacification anormalenaent développée de $\Gamma$ arborisation

11 Gastroenterología, Vol. 97, No. 2 (1962)

130

Editorial

intra-hépatique, il est coutume d'admettre une repercussion de la stase en aval. En effet, il n'existe pas d'affectíon primitive des rameaux bílíaíres íntra-hépatiques sans dilatation du canal hépato-cholédoque, affirme J. Caroli dans un bel article (Médecine et Hygiene; vol. 19, p. 867869, 15 nov. 1961) consacré à ce problème curieusement nou-veau. Le chirurgien devra done se garder d'accuser systématique-nient la region papillaire, sans se préoccuper de ce qui peut se passer au-dessus, en plein parenchyme du foie.

Caroli limite son sujet aux collecteurs segmentaíres d'un certain dia-mètre, excluant les capillicules biliaires, cholangioles de l'espace porte et autres vaisseaux microscopiques. II precise d'abord les notions essentielles de radio-anatomie des voies biliaires intra-bépatiques explorées par cholangiographie veineuse; elles font intervenir le blocage du sphincter d'Oddi par la morphine (pour éviter une evacuation trop précoce de la bile iodée); le centrage correct des films et les positions spéciales données au malade; la tomographie; et le tirage des cliches au Logetron. Un remplíssage précoce, ímmédiat et complet des voies biliaires intra-hépatiques est toujours le sígne d'une stênose sous-jacente, dont il faudra determiner le niveau.

$* * *$

A côté des dilatations secondaires à des tumeurs (malignes ou bénignes), à des kystes (simples ou parasitaires), on rencontre essen-tiellement trois états pathologiques des voies biliaires intrahépatiques :

I. Les dilatations polykystíques, congénitales (sans relation avec le «foie polykystique», qui n'est pas de structure biliaire), dans lesquelles les ectasies segmentaires, non exclues, contiennent de la bile et sont à $\Gamma$ origine d'accès angiocholitiques ou lithiasiques. Cliniquement, elles sont caractérisées par des poussées fébriles d'évolution capricieuse, ayant commence dès l'enfance et sans retention ictérique; les douleurs sont facultatives, liées à des thromboses calculeuses secondaires. L'angiocholégraphie révèle l'existence d'une série de taches opaques disséminées surtout à la périphérie du foie.

II. La papíllomatose est rarement diagnostiquée au niveau des voies biliaires intra-hépatiques. Elle se traduit par des accès angio cholitiques avec ictère, et parfois hémobilie. Radiologiquement, les énormes dilatations sont encombrées par des lacunes irrégulières.

III. La lithíase intra-hépatique, fréquente en Extreme-Orient (à 
Editorial

131

cause de la participation parasitaire) est exceptionnelle chez nous; les crises, classiques, s'accompagnent d'un mauvais état general, sans hépatomégalie. A Гintervention, on palpe une série de tumeurs duГes dans le foie, et la cholangiographie per-opératoire retrograde donne une image en «pince de homard» dirigée vers le haut, avec absence de remplissage monolobaire.

$* * *$

La thérapeutíque chirurgicale de ces états est, dans $\Gamma$ ensemble, assez décevante, par Гimpuissance d'abord, la gravité d'autre part de Гintervention. Les cavités polykystiques intra-parenchymateuses sont inabordables. L'hépatectomie réglée peutparfois guérir unepapillomatose lobaire; elle atteint rarement $\Gamma$ ensemble des concretions lithiasiques, et doit être complétée, dans ce cas, par une anastomose cholédoco-jéjunale. M. D.

Seite der Therapie - Page de thérapeutique

Crísmer, R.; Ramíoul, H. et Dreze, Ch.: Le traitement de la rectocolite ulcéro-hémorragique par la radiothérapïe diencéphalique. Acta Gastro-ent. belg. 24: 507-521 (août/sept. 1961).

Les auteurs ont traité par la radiothérapie diencéphalique douze cas de rectocolite ulcérohémorragique, obtenant un tiers d'excellents resultats immediate. Ces resultats sont done comparables à ceux obtenus au moyen d'autres therapeutiques réputées actives dans cette redoutable affection dont on connaît revolution capri-cieuse et imprévisible.

Cependant la radiothérapie diencéphalique agit sur Гintestin, probablement par voie neurohumorale. La stimulation des noyaux hypothalamiques, agissant indi-rectement sur Thypophyse, pourrait provoquer une liberation d'ACTH, induisant à son tour une secretion accrue d'hormones corticosurrenaliennes. Les epreuves biologiques effectuées paraissent confirmer cette hypersécrétion d'ACTH et tout se passe comme si la radiothérapie diencéphalique agissait en déclenchant une «auto-hormonothérapie».

Les doses faibles utilisées au cours de la radiothérapie diencéphalique paraissent sans danger, mais il faut éviter d'irradier les patients qui ont des antecedents neurologiques.

Kayabalí, I.: Quelques indications de la neurectomie péri-artère hépatique. Lyon Chir. 56: 857864 (1960).

Depuis 1948, la neurectomie péri-artère hépatique de Mallet-Guy a été mise en pratique et le mode d'action de cette intervention peut se résumer comme anti-lipopexique, anti-inflammatoire et vasodilatateur. 